\title{
Conformational Space Annealing by Parallel Computations: Extensive Conformational Search of Met-enkephalin and of the 20-Residue Membrane-Bound Portion of Melittin
}

\author{
JOOYOUNG LEE, HAROLD A. SCHERAGA \\ Baker Laboratory of Chemistry and Chemical Biology, Cornell University, Ithaca, New York 14853-1301
}

Received 3 November 1998; revised 4 January 1999; accepted 12 January 1999

\begin{abstract}
A successful implementation of a parallel version of a conformational space annealing (CSA) method is presented. The CSA method, an optimization procedure for conformational energy calculations on polypeptides, searches the whole conformational space in its early stages and then narrows the search to smaller regions with low energy and distinct geometry. By selecting 20 seed conformations that are far from each other in the conformational space, the CSA method generates up to 400 conformations to be minimized independently, thereby allowing very efficient parallel computations to be carried out. When applied to the pentapeptide Met-enkephalin, the global minimum-energy conformation (GMEC) of the ECEPP / 3 (Empirical Conformational Energy Program for Peptides) force field was found for all of 600 separate runs after about $35.5 \mathrm{~s}$ each, on average, of wall clock time, using 16 processors of an IBM SP2 supercomputer. The GMEC of the 20-residue membrane-bound portion of melittin was also found for all of 24 independent runs. The average wall clock time to find the GMEC of this 20-residue peptide, using 32 processors of an IBM SP2 supercomputer, was about $4.5 \mathrm{~h}$ per run. (c) 1999 John Wiley \& Sons, Inc. Int J Quant Chem 75 : 255-265, 1999
\end{abstract}

Key words: protein folding; conformational space annealing; global minimum-energy conformation; ECEPP/3; parallel computation 


\section{Introduction}

$\mathbf{F}$ olding a protein by computer simulations using only its sequence information is a current fundamental problem. The difficulty of the protein-folding problem is that it consists of two separate and very hard problems: namely, obtaining an appropriate potential function and developing an efficient optimization method to search the conformational space of a given protein sequence. Considering the fact that no such potential functions that can fold proteins into native structures utilizing only their sequence information yet exist, it is very important to develop a powerful optimization method which can search the entire conformational space of a given protein sequence efficiently. Only after one can search the entire conformational space can the potential function be fine-tuned and tested for its validity for the study of protein folding. This article pertains to such an optimization method.

Recently, we developed a very powerful optimization method for protein folding, which we designate as a conformational space annealing (CSA) method. It was tested on Met-enkephalin, a pentapeptide with 24 dihedral angles [1], and on the 20-residue membrane-bound portion of melittin with 113 variable dihedral angles [2]. In this article, we propose a very efficient implementation of a parallel version of the CSA method and provide an extensive investigation of the conformational searches for these two peptides. We demonstrate that the global minimum-energy conformation (GMEC) of the ECEPP / 3 force field [3-6] (Empirical Conformational Energy Program for Peptides) for the two peptides can be obtained within a fraction of $1 \mathrm{~min}$ and a few hours of wall clock time, respectively, using 16-32 processors of an IBM SP2 supercomputer.

The essence of the CSA method relies on the realization of the difficulty of the problem: For a given potential function, it is quite likely that there exist many distinct low-lying local energy minima which are far from each other in the conformational space. Therefore, it is necessary to consider an optimization method that should deal with not one but many conformations simultaneously and also that should cover the whole conformational space. The current version of the CSA method searches the whole conformational space in its early stages and then narrows the search to smaller regions with low energy.

\section{Method}

We first present a brief summary of the CSA algorithm [1,2] and then describe more details of the algorithm including those changes that are necessary for efficient implementation of parallel computations with the CSA method. A flow chart of the algorithm is presented in Figure 1.

\section{OVERVIEW OF CSA}

As in genetic algorithms [7, 8], CSA starts with a random population of conformations. In addition, we consider only the conformational space of local minima: All conformations are energy-minimized with a local minimizer such as SUMSL [9] (Secant Unconstrained Minimization Solver). The algorithm starts by generating 50 (this is an arbitrarily large number) random conformations which are then minimized with SUMSL. We call these 50 conformations the first bank. It should be noted that conformations in the first bank remain unchanged throughout the rest of the algorithm and are augmented in number when necessary (see the subsection Criterion to Add More Conformations to the Bank). Figure 2 is a schematic representation of the local minima in the conformational space and the 50 randomly selected conformations that constitute the first bank. We copy the first bank and save it as the bank.

We then select 20 dissimilar conformations from the bank, excluding those that (in subsequent iterations) have already been used; they are called seeds (for more details on the selection of seeds, see subsection Selection of Seeds). Each seed conformation is modified by replacing some of its dihedral angles with those from the remaining 49 conformations in the bank (see the subsection Trial Conformations) to generate 20 conformations (a total of 400 for 20 seeds) which are again energyminimized with SUMSL. The level of modification of a seed varies from substituting one dihedral angle to substituting up to one-third of the total number of dihedral angles pertaining to a contiguous portion of the peptide; the new values of the dihedral angles are chosen from the other 49 conformations in the bank. These minimized conformations are called trial conformations. Each trial 


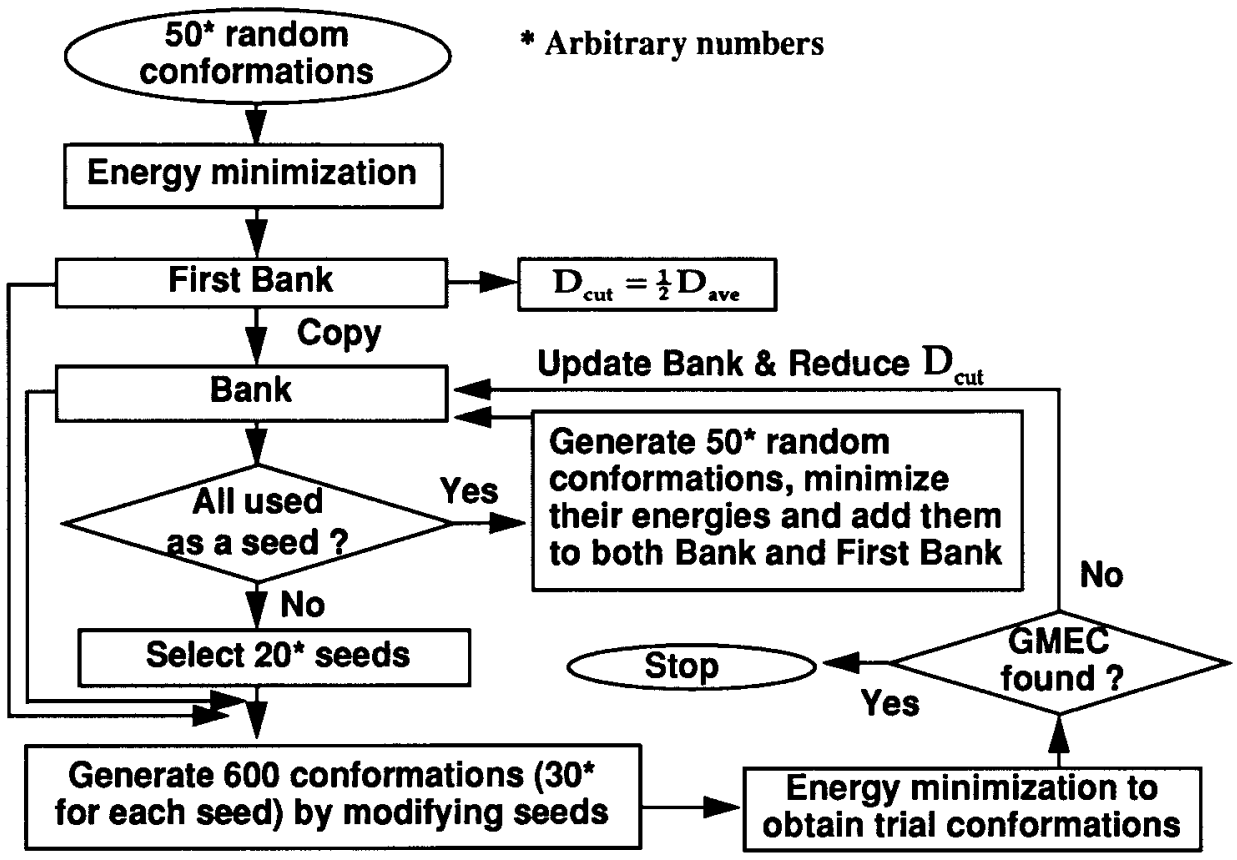

FIGURE 1. Flow chart of the CSA algorithm.

conformation is used to update the bank along with the length scale $D_{\text {cut }}$, which approximately sets the distance between two conformations in the bank. Narrowing the search region is accom-

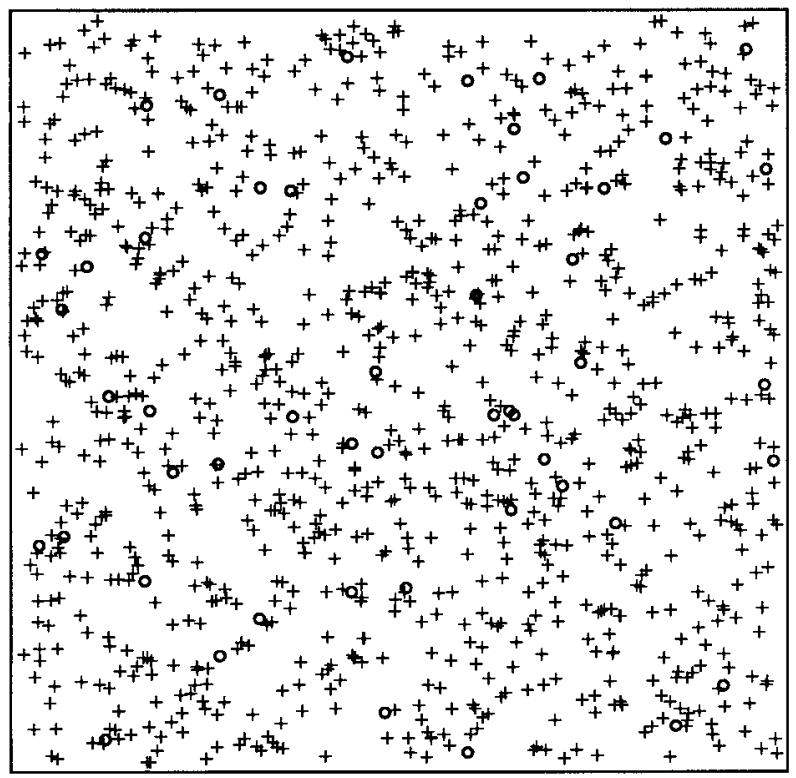

FIGURE 2. Schematic representation of conformational space. The plus signs indicate unexplored local minima, and the 50 open circles represent the conformations that constitute the initial bank (defined as the first bank in the text). plished by setting $D_{\text {cut }}$ to a large value initially and gradually reducing it. For more details on updating the bank with trial conformations, see the subsection Update the Bank with Trial Conformations. The narrowing of the conformational search is illustrated schematically in Figure 3. The procedure for selecting another set of 20 seeds and generating 400 trial conformations, to update the bank further, is iterated until all 50 conformations in the bank have already been used as a seed, completing one round of the CSA procedure. This round is repeated for a predetermined number of times. If the conformational search is not successful (e.g., if the known GMEC is not yet obtained), more random conformations are added to the bank (beyond 50) and the whole procedure is repeated. (If the GMEC is not known in advance, the CSA algorithm will keep adding more random conformations to the bank after a predetermined number of rounds have been repeated.)

\section{ELIMINATION OF DIHEDRAL ANGLES IN UNPHYSICAL REGIONS IN THE FIRST BANK}

Although it is not essential, in this work, we added a procedure that helps to eliminate some values of dihedral angles that occur in unphysical regions in the first bank. The first bank is the collection of random energy-minimized conforma- 


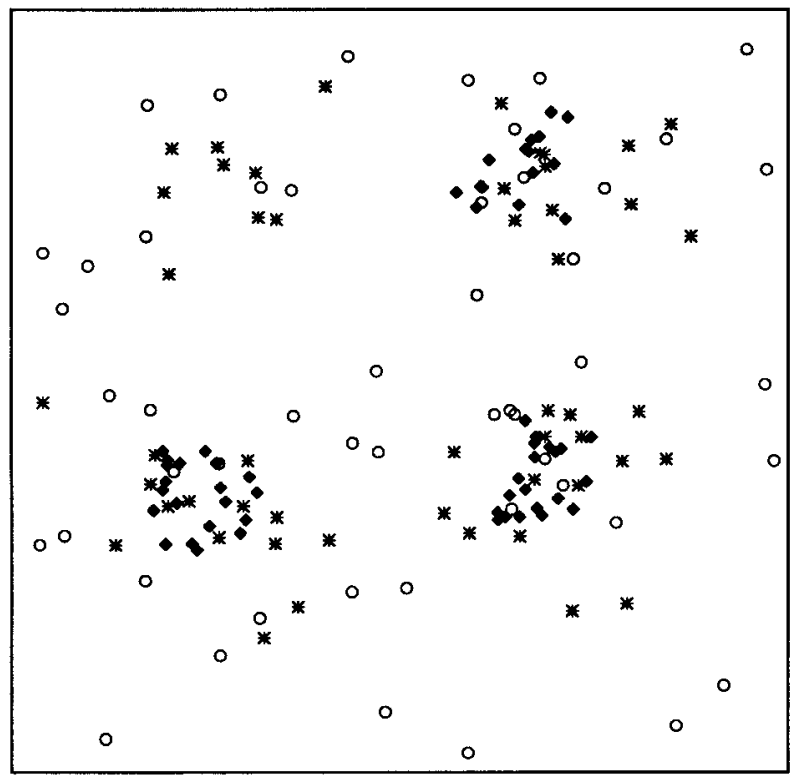

FIGURE 3. Schematic diagram to illustrate the evolution of the bank conformations as the CSA algorithm proceeds. The 50 open circles are the same as in Figure 2 representing the first bank. The 50 stars represent the bank conformations at an intermediate stage of CSA. It should be noted that the conformations are now more clustered into smaller regions. Fifty conformations in the late stages of CSA are represented as diamonds, which are even more densely clustered.

tions, which is the bank at the very beginning of CSA. The information stored in the first bank is used as the random variables in CSA [1, 2]. For example, the dihedral angles $\omega$ are not searched randomly from $-180^{\circ}$ to $180^{\circ}$, but only from the values of $\omega$ in the first bank which are mostly near either $180^{\circ}$ (trans) or $0^{\circ}$ (cis). As the size of a peptide increases, random energy-minimized conformations are more likely to contain values of dihedral angles that are unphysical, e.g., with $\omega$ near $\pm 90^{\circ}$ or $|\phi|<40^{\circ}$, etc. If this happens, modifications of dihedral angles of random energyminimized conformations are attempted only a few times by substituting allowed values for the unphysical ones and then reminimizing the energies of these conformations. It should be noted that these attempts are not meant so much to eliminate all of the unphysical values of dihedral angles, but to reduce their number with minimal effort. The following are the modifications that are introduced at this stage:

1. For $20^{\circ}<|\omega| \leq 90^{\circ}, \omega$ is set to $0^{\circ}$, and for $90^{\circ}<|\omega| \leq 160^{\circ}, \omega$ is set to $180^{\circ}$.
2. For $\left|\chi^{1}\right| \leq 30^{\circ}$, and $90^{\circ}<\left|\chi^{1}\right| \leq 150^{\circ}$, the values of $\chi^{1}$ are randomly set to either $30^{\circ}<$ $\left|\chi^{1}\right| \leq 90^{\circ}$, or $150^{\circ}<\left|\chi^{1}\right| \leq 180^{\circ}$. This reduces the nonbonded repulsion between atoms attached to $\mathrm{C}^{\alpha}$ and those attached to $\mathrm{C}^{\beta}$.

3. The following regions of $(\phi, \psi)$ of all residues except the first and the last residue of a peptide are randomly set to the values outside the corresponding regions:
(a) $90^{\circ}<\psi \leq 140^{\circ}$ with $\phi>0$.
(b) $-140^{\circ}<\psi \leq-90^{\circ}$ with $\phi<0$.
(c) $|\phi| \leq 40^{\circ}$.
(d) For all residues except glycines, $\phi>0$ with $\psi<0$.

\section{SELECTION OF SEEDS}

In CSA, special attention is paid to cover as much of conformational space as possible by introducing two key procedures. The first one is to select seed conformations that are far from each other in conformational space. The second procedure is discussed in the subsection Update the Bank with Trial Conformations. Each seed conformation is modified at various stages to generate many trial conformations that are, to some extent, similar to each other, that is, close to the seed conformation in conformational space. Therefore, it is important not to select seeds that are close in conformational space.

To achieve this goal, the following steps are carried out to select $n$ seeds ( $n=20$ is used in Fig. 1 ): We first consider the case in which $n<m$, where $m$ is the number of conformations that can be selected as seeds. (Initially, $m$ is equal to the number of conformations in the bank, say 50. But, in later stages, the $50-m$ conformations in the bank have already been used as seeds and are not selected as seeds until all conformations in the bank are used.) The first seed $s_{1}$ is selected randomly (not necessarily as the lowest-energy one) out of $m$ conformations, leaving $m-1$ conformations available as future seeds.

All the distances between the seed $s_{1}$ and the $m-1$ conformations are measured according to the following equation [1]:

$$
\begin{aligned}
D_{i j}= & \sum_{k=1}^{n} \min \left[\bmod \left\{\left(\theta_{k}^{i}-\theta_{k}^{j}\right), \operatorname{sym}(k)\right\},\right. \\
& \left.\left\{\operatorname{sym}(k)-\bmod \left\{\left(\theta_{k}^{i}-\theta_{k}^{j}\right), \operatorname{sym}(k)\right\}\right\}\right],
\end{aligned}
$$


where $\bmod (A, B)$ is the least positive value of $x$ satisfying $A=l B+x$ with an integer $l$, and $\operatorname{sym}(k)$ is $360^{\circ}, 180^{\circ}$, and $120^{\circ}$ for the dihedral angle $k$ with no symmetry, twofold symmetry, and threefold symmetry, respectively. The distance $D_{i j}$ between two conformations $i$ and $j$ is defined as the sum of the differences of each dihedral angle in conformations $i$ and $j$, respectively. Considering only those conformations that are further away from $s_{1}$ in conformational space than $d_{\text {ave, }}$, the average distance between $s_{1}$ and the $m-1$ conformations, the second seed $s_{2}$ is selected as the lowest-energy conformation, leaving $m-2$ conformations now available as seeds. A schematic diagram to illustrate this procedure is shown in Figure 4. By defining the distance between a set of already selected seeds $\left(s_{1}, s_{2}\right)$ and a conformation $i$ from the $m-2$ conformations as the minimum of two distances between each seed in the set and the conformation $i$, all the distances between $\left(s_{1}, s_{2}\right)$ and $m-2$ conformations are measured according to Eq. (1). Again considering only those conformations that are further away from $\left(s_{1}, s_{2}\right)$ than $d_{\text {ave }}$, now the average distance between $\left(s_{1}, s_{2}\right)$ and $m-2$ conformations, the third seed $s_{3}$ is selected as the lowest-energy conformation, which now leaves $m-3$ conformations available as seeds. A similar procedure is repeated, one by one, to select all $n$ seeds. It should be noted that conformations close to the already selected seeds are not likely to be chosen as seeds. If $n=m$, all $m$ conformations are selected as seeds.
We now consider the case $n>m$. First, all $m$ conformations are selected as seeds. The next seed is selected randomly considering all 50 conformations in the bank, and subsequent seeds are selected, one by one, by following the same procedure as described above for $n<m$, considering all 50 conformations in the bank.

Selecting many seeds to generate a large number of trial conformations does not allow as frequent updating of the bank as in the earlier version of CSA $[1,2]$, which may slow down the performance of CSA. However, the procedure of selecting seeds which are far from each other in conformational space helps to increase the diversity in trial conformations-consequently, in the bank conformations-as compared to the earlier version of CSA in which only one seed was selected for generation of trial conformations.

\section{TRIAL CONFORMATIONS}

Each seed conformation is used to generate trial conformations from it by replacing some of its dihedral angles with those from one of the remaining conformations in the bank, according to the following procedure: In generating trial conformations from each seed, we followed the same procedure as described in our earlier work $[1,2]$. In the present work, however, we treated the backbone dihedral angles $(\phi, \psi)$ of nonglycine residues as one variable to substitute as a pair rather than two variables to substitute individually. The dihedral

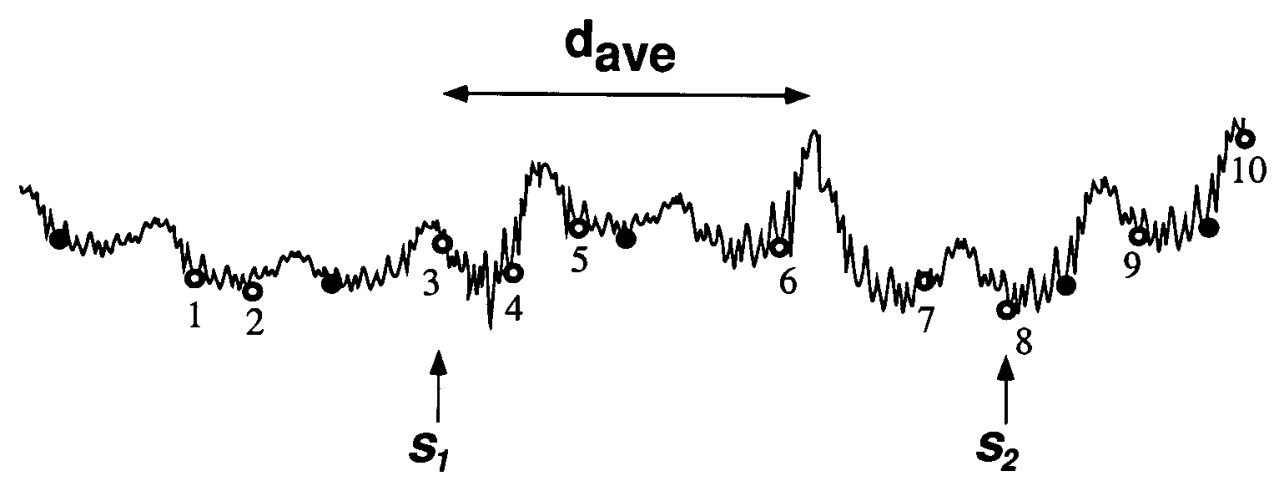

FIGURE 4. Schematic diagram to illustrate the procedure to select 2 seeds from the bank conformations. The landscape of a complex energy function is represented schematically as a one-dimensional curve. Conformational space and its corresponding energy correspond to the $x$ and $y$ axes, respectively. The current (local minima) bank conformations (of size 15) are represented as five filled circles (those already used as seeds) and 10 open circles (those that have not yet been used as seeds and, hence, available as seeds). The first seed $s_{1}$ is selected randomly out of the 10 open circles. The average distance $d_{\text {ave }}$ between $s_{1}$ and the rest of the nine open circles is measured. The second seed $s_{2}$ is selected as the lowest-energy conformation among the four open circles $(7,8,9$, and 10$)$ that are further away from $s_{1}$ than $d_{\text {ave }}$. 
angles $\phi$ and $\psi$ of glycine residues are treated individually. The procedure to generate 20 trial conformations for a given seed is as follows (for a 20-residue peptide):

1. Generate six conformations by replacing a few (one to five) randomly selected dihedral angles of the seed conformation with corresponding ones in the first bank.

2. Generate six conformations by replacing a few (one to five) randomly selected dihedral angles [but only from $\phi, \psi$, and $\chi^{1}$ (for residues with more than one side-chain dihedral angle)] of the seed conformation with corresponding ones in the first bank.

3. Generate three conformations by replacing a group of dihedral angles which are either all side-chain dihedral angles of a residue (if it has more than one side-chain dihedral angle) or all backbone dihedral angles plus $\chi^{1}$ of a residue with corresponding ones in the bank.

4. Generate five conformations by replacing all dihedral angles pertaining to a contiguous portion of size four to eight residues of the peptide with corresponding ones in the bank.

For the peptapeptide Met-enkephalin, 10 trial conformations for each seed (three, three, two, and two for each step above) were generated similarly. For the last step, contiguous portions of size one to three residues were used. It should be noted that we use the first bank (not the bank) for the first two steps. The reason is that dihedral angles in the first bank are used as replacements for random variables, whereas those in the bank are updated in subsequent stages in the CSA algorithm. All conformations are subsequently energy-minimized with SUMSL [9].

\section{UPDATE THE BANK WITH TRIAL CONFORMATIONS}

The second key procedure in CSA is to introduce a length scale $D_{\text {cut }}$, which sets the approximate allowed distance between two conformations in conformational space [1, 2]. The diversity of the bank conformations can be controlled by changing the value of the cutoff distance $D_{\text {cut }}$. In CSA, the cutoff distance $D_{\text {cut }}$ is set to a large value at the beginning of the algorithm (for searching the whole conformational space) and then is reduced slowly to a smaller value (for narrowing the search to smaller regions of low-energy conformations). This is accomplished by updating the bank with each trial conformation $\alpha$ (generated as described in the subsection Trial Conformations) as follows:

First, find the conformation $A$ in the bank which is the closest to one of the trial conformations $\alpha$ at a distance $D_{\alpha A}$. If $D_{\alpha A}<D_{\text {cut }}, \alpha$ is considered similar to $A$; in this case, $\alpha$ replaces $A$, if, in addition, it is lower in energy. If $\alpha$ is not similar to $A$ (i.e., $D_{\alpha A}>D_{\text {cut }}$ ), but its energy is lower than that of the highest-energy conformation in the bank, $B, \alpha$ replaces $B$. If neither of the above conditions holds, $\alpha$ is rejected. Thus, the number of conformations in the bank remains unaltered. A schematic diagram illustrating this procedure is shown in Figure 5. After the bank is updated with all trial conformations, the value of $D_{\text {cut }}$ is reduced according to the following schedule $[1,2]$ : The initial and final value of $D_{\text {cut }}$ is set, respectively, to one-half and one-fifth of the average pair distance in the first bank, $D_{\text {ave }}$. The value of $D_{\text {cut }}$ is reduced in steps [from $(1 / 2) D_{\text {ave }}$ to $(1 / 5) D_{\text {ave }}$ ] in 2000 (or $10,000)$ minimizations for Met-enkephalin (or for a 20-residue peptide).

The procedure for selecting a set of seeds, generating trial conformations, and updating the bank is iterated until all conformations in the bank have already been used as a seed, completing one round of the CSA procedure. The bank used at the start of the next round is the one that was obtained at the end of the immediately preceding round. Several rounds are implemented for a predetermined number of times.

\section{PARALLEL CSA}

The local energy minimization of trial conformations (typically 20 for a seed conformation in [1, $2]$ is the most time-consuming part of the algorithm (over $99 \%$ of the CPU time), and each minimization is independent of the others. Therefore, it is straightforward to program CSA in a parallel environment. Using 20 seeds at a time, we were able to generate 400 conformations (20 for each seed) which were subsequently energy-minimized. This enabled us to use many processors simultaneously. With 400 conformations, one can use, in principle, up to 401 processors (one processor to distribute and collect data and 400 processors to minimize the energy of one conformation for each processor). However, with 401 processors, the efficiency of parallel computing (defined here as the ratio of the CPU time using one processor over the 


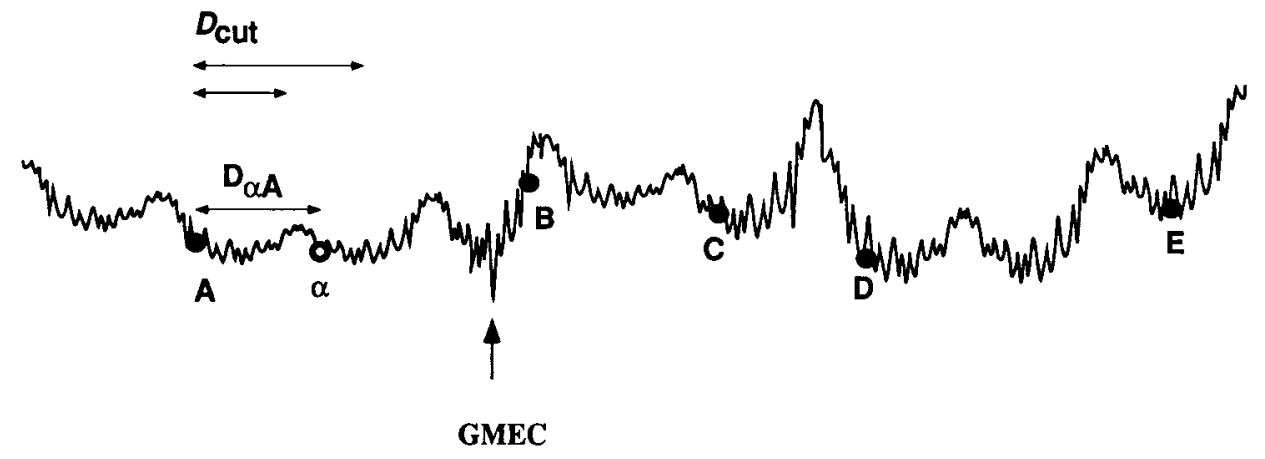

FIGURE 5. Schematic diagram to illustrate the procedure to update the bank conformations with a trial conformation $\alpha$ generated as described in subsection Trial Conformations. The bank conformations (of size 5 in this example) are labeled with capital letters. First, by measuring all the distances between the trial conformation $\alpha$ and the bank conformations $A, B, C, D$, and $E$, find the closest conformation $A$ (to $\alpha$ ) at a distance $D_{\alpha A}$. The procedure to update the bank depends on the relative size of $D_{\alpha A}$ and $D_{\text {cut }}$. If $D_{\alpha A}<D_{\text {cut }}$ (with the larger size of $D_{\text {cut }}$ shown in the figure), $\alpha$ replaces $A$ if, in addition, $\alpha$ is lower in energy than $A$. However, if $D_{\alpha A}>D_{\text {cut }}$ (with the smaller size of $D_{\text {cut }}$ shown in the figure), $\alpha$ replaces $B$, the highest-energy (most unfit) conformation in the bank, if $\alpha$, in addition, is lower in energy than $B$. If $\alpha$ does not satisfy the "lower in energy" condition in either of the two cases, $\alpha$ is discarded.

total combined wall clock time of all processors to complete the identical computation) can decrease to considerably less than $50 \%$. This is because the CPU time to complete one local energy minimization depends on the details of the input conformation, and until the minimization that takes the most CPU time is completed, each of the other processors has to wait after its own minimization is completed. The efficiency can be improved by using a smaller number of processors to reduce the waiting time. We achieved over $80 \%$ efficiency by using 32 (16) processors with 400 (200) trial conformations. One processor took care of the distribution and collection of conformations and the others were assigned to complete local energy minimization of the incoming conformations.

\section{CRITERION TO ADD MORE CONFORMATIONS TO THE BANK}

For complex systems like proteins, it is possible that the CSA method may fail to find the GMEC after completing the narrowing of the search to smaller regions of low-energy conformations. This situation may arise for various reasons such as the total number of bank conformations (which was set arbitrarily) is too small or the annealing schedule for $D_{\text {cut }}[1,2]$ was set too fast. One way to solve this problem is to add more random conformations to the bank and repeat the CSA procedure $[1,2]$.

The main iteration of CSA shown in Figure 1 (selecting seeds, modifying them to generate trial conformations whose energies are minimized, and updating the bank with these trial conformations) is repeated until all of the bank conformations are used as seeds. This corresponds to the case $m=0$ in the subsection Selection of Seeds, and one round of iteration is completed. Compared to our previous work [1,2], in which a single seed was used to generate trial conformations, it took a considerably larger number of minimizations here to finish one round of iteration. In the present work, we used a maximum of four rounds of iteration before increasing the size of the bank by adding new random minimized conformations. The first round always took a considerably larger number of minimizations than did the subsequent rounds. Although different criteria for adding more conformations to the bank were employed in $[1,2]$, the essence of these criteria is to determine approximately when the bank conformations are not producing any new conformation after the search in conformational space has been narrowed to smaller regions. If this happens, it is necessary to increase the size of the bank by adding more random minimized conformations to search other regions of conformational space.

\section{Results}

First, we applied the parallel version of CSA to Met-enkephalin, a pentapeptide with 24 dihedralangle degrees of freedom. For all of 600 indepen- 
dent runs (300 with 10 seeds and another 300 with 20 seeds for each iteration), the accepted GMEC was obtained after only about 2420 local minimizations, corresponding to 181,000 energy evaluations, which are similar to previous CSA results with a single seed [1]. The wall clock time to obtain the GMEC was about $35.5 \mathrm{~s}$ each, on average, with 16 processors of the IBM SP2 supercomputer at the Cornell Theory Center. The scatter plot of the number of function evaluations for all 600 runs is shown in Figure 6. On average, it took more function evaluations with 20 seeds $(188,000$ compared to 175,000$)$. However, the average wall clock time for runs with 20 seeds was less than that with 10 seeds (32.4 compared to 38.6 seconds), demonstrating that the efficiency of parallel computation increases as the number of trial conformations increases.

A summary of the results for the 20-residue membrane-bound portion of melittin is presented

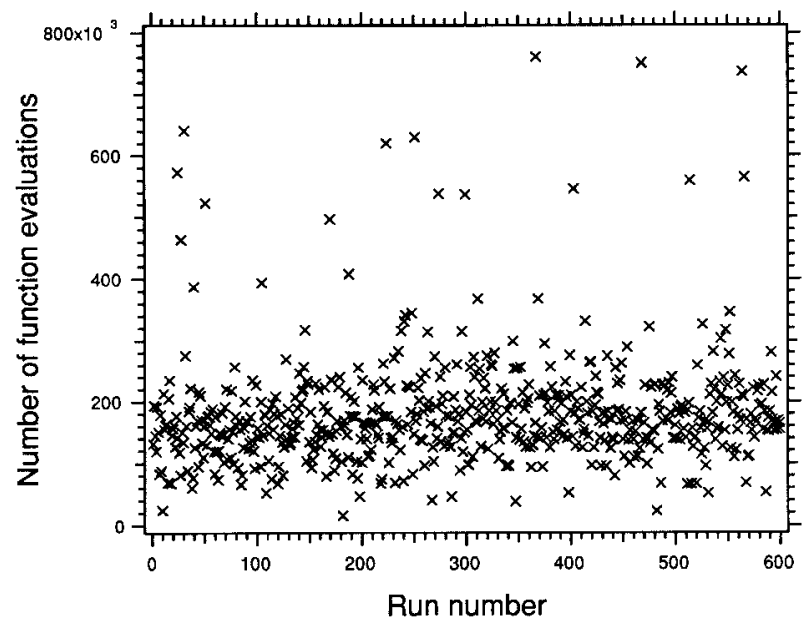

FIGURE 6. Scatter plot of the number of function evaluations for 600 independent runs to obtain the accepted GMEC of Met-enkephalin with energy $E=$ $-11.707 \mathrm{kcal} / \mathrm{mol}$ in each run. The GMEC was obtained successfully for all 600 independent runs with about 181,000 function evaluations corresponding to about 2,420 local energy minimizations on average. The average wall clock time to obtain the GMEC took about $35.5 \mathrm{~s}$ with 16 processors of an IBM SP2 supercomputer. The first 300 points correspond to the runs with 10 seeds and the next 300 corresponds to the runs with 20 seeds. On average, it took more function evaluations with 20 seeds (188,000 compared to 175,000). However, the average wall clock time for runs with 20 seeds was less than those with 10 seeds ( 32.4 compared to $38.6 \mathrm{~s}$ ), demonstrating that the efficiency of parallel computation increases as the number of trial conformations increases. in Table I. There are 113 variable dihedral angles in the peptide. In 24 independent runs (14 runs with 10 seeds and 10 runs with 20 seeds), the proposed GMEC [2] of the peptide was obtained in all cases after a total of about 1.2 million combined minimizations. All 24 runs were programmed to terminate the search if a conformation with energy less than $-91.0 \mathrm{kcal} / \mathrm{mol}$ was found, and the lowest-energy conformation from each run was identical to the proposed GMEC [2] with energy $-91.02 \mathrm{kcal} / \mathrm{mol}$. In a recent investigation of the same peptide by the new electrostatically driven Monte Carlo (EDMC) method [10], the identical GMEC was found in four out of 14 independent runs after over 1.1 million minimizations were carried out. It should be noted that previous investigations of the same peptide by various methods such as the buildup procedure [11], the earlier EDMC method [12], and the self-consistent multitorsional field method [13] failed to locate the GMEC [2]. The values of the parameters used in CSA for Met-enkephalin and the 20-residue membrane-bound portion of melittin are shown in Table II.

In Figure 7, real data (for Leu-13) are shown to validate the illustration in Figure 3. The data for the 20-residue membrane-bound portion of melittin were collected at the beginning of a CSA run, in the middle, and when the GMEC was obtained. The values of $(\phi, \psi)$ of Leu-13 at the GMEC are $\left(-147^{\circ}, 75^{\circ}\right)$. Although Figure 7 is only a projection of the conformational space in two variables $(\phi, \psi)$ of Leu-13 while Figure 3 represents the whole conformational space, indeed, the CSA method appears to cover the whole conformational space at its early stages and narrows the conformational search to smaller regions of low-energy local minima. In this 20-residue peptide, the Leu-13 residue precedes Pro-14.

\section{Discussion}

The successful implementation of a very efficient version of parallel CSA is based on the selection of many seed conformations at a time which, consequently, generates a large number of trial conformations to be energy-minimized. Generating a large number (400) of trial conformations means less frequent updating of the bank than the earlier version of CSA [1, 2], in which a much smaller number of trial conformations (20) was generated. 
TABLE I

Summary of the 24 runs carried out on the 20 -residue membrane-bound portion of melittin. ${ }^{\text {a }}$

\begin{tabular}{|c|c|c|c|c|}
\hline $\begin{array}{l}\text { Run } \\
\text { no. }\end{array}$ & $\begin{array}{c}\text { No. } \\
\text { minimizations }\end{array}$ & $\begin{array}{c}\text { No. } \\
\text { evaluations }\end{array}$ & $\begin{array}{l}\text { Wall clock time with } \\
32 \text { processors (h) }\end{array}$ & $\begin{array}{l}\text { Final size } \\
\text { of bank }\end{array}$ \\
\hline 1 & 70,150 & $16.9 \times 10^{6}$ & 6.3 & 150 \\
\hline 2 & 87,400 & 21.1 & 8.0 & 200 \\
\hline 3 & 38,900 & 9.4 & 3.6 & 100 \\
\hline 4 & 21,250 & 5.4 & 2.0 & 50 \\
\hline 5 & 35,500 & 8.2 & 3.1 & 100 \\
\hline 6 & 22,050 & 5.3 & 2.0 & 50 \\
\hline 7 & 15,650 & 3.8 & 1.4 & 50 \\
\hline 8 & 40,700 & 9.6 & 3.6 & 100 \\
\hline 9 & 35,100 & 8.2 & 3.1 & 100 \\
\hline 10 & 83,750 & 19.4 & 7.4 & 150 \\
\hline 11 & 45,300 & 10.6 & 4.0 & 100 \\
\hline 12 & 79,150 & 18.8 & 7.1 & 150 \\
\hline 13 & 78,550 & 18.1 & 6.9 & 150 \\
\hline 14 & 39,300 & 9.3 & 3.5 & 100 \\
\hline 15 & 25,250 & 7.0 & 2.4 & 50 \\
\hline 16 & 23,650 & 6.6 & 2.3 & 50 \\
\hline 17 & 26,850 & 7.2 & 2.5 & 50 \\
\hline 18 & 53,700 & 14.2 & 4.9 & 100 \\
\hline 19 & 46,100 & 12.1 & 4.2 & 100 \\
\hline 20 & 62,900 & 16.4 & 5.7 & 100 \\
\hline 21 & 130,600 & 33.4 & 11.9 & 200 \\
\hline 22 & 58,900 & 14.7 & 5.1 & 100 \\
\hline 23 & 49,300 & 13.1 & 4.5 & 100 \\
\hline 24 & 14,450 & 4.2 & 1.4 & 50 \\
\hline Ave. & 49,352 & 12.2 & 4.5 & \\
\hline
\end{tabular}

${ }^{a}$ In the first 14 runs, 10 seeds were selected for each iteration to generate 200 trial conformations. In the next 10 runs, 20 seeds were used to generate 400 conformations to be energy-minimized. The total number of minimizations for all 24 runs is about 1.2 million. The GMEC of the peptide was obtained for all 24 independent runs. The average wall clock time, using 32 processors of an IBM SP2 supercomputer, was $4.5 \mathrm{~h}$.

(The frequency of bank updating is determined by the number of trial conformations generated for each iteration as shown in Fig. 1.) This may slow down the performance of CSA. However, selecting many seed conformations that are far from each other in conformational space, as described in this article, helps to increase the diversity of the bank conformations as compared to the earlier version of CSA with one seed. We believe that the increased diversity of the bank compensates for the disadvantage of less frequent updating of the bank.

In this article, we have successfully implemented the parallel version of CSA and demonstrated that the GMEC of a pentapeptide can be obtained within a fraction of 1 minute of wall clock time using 16 processors of an IBM SP2 supercomputer. The corresponding wall clock time for a 20-residue peptide using 32 processors is of the order of a few hours. It should be noted that the GMEC of the 20-residue membrane-bound portion of melittin was found with $100 \%$ success for all 24 independent runs. This implies that a successful conformational search of up to a 40- to 50-residue peptide is a possibility by parallel CSA computations; this would enable us to test and fine-tune existing potential functions for protein folding by directly comparing experimental structures of small proteins with those obtained by CSA. This method has also been applied to larger proteins [14, 15].

It should also be noted that the greatest advantage of the CSA method is that it always finds distinct families of low-energy conformations [1, 2]. This is accomplished in CSA by searching the 
TABLE II

Details of CSA parameters of Met-enkephalin and 20-residue membrane-bound portion of melittin.

Met-enkephalin

20-Residue membrane-bound portion of melittin

No. residues

No. variable dihedral angles

Initial size of bank ${ }^{\mathrm{a}}$

Average no. energy evaluations per minimization

$D_{\text {ave }}$ of the first bank

Initial value of $D_{\text {cut }}$

Terminal value of $D_{\text {cut }}$

No. minimizations used in annealing

Round limit ${ }^{\mathrm{C}}$

No. trial conformations per seed

No. seeds

No. trial conformations per iteration

5

5
24

50

75

$1350^{\circ}$

$(1 / 2) D_{\text {ave }}{ }^{\mathrm{b}}$

$(1 / 5) D_{\text {ave }}$

2000

4

10

10,20

100,200
20

113

50

247

$5600^{\circ}$

(1/2) $D_{\text {ave }}$

$(1 / 5) D_{\text {ave }}$

10,000

4

20

10,20

200, 400

\footnotetext{
${ }^{a}$ The final size of the bank for Met-enkephalin was 50 for 594 runs and 100 for six runs. The final size of the bank for the 20 -residue peptide ranged from 50 to 200 .

${ }^{\mathrm{b}}$ The value of $D_{\text {ave }}$ is the average pair distance in the first bank.

${ }^{c}$ Round limit is the maximum number of rounds of iterations allowed before adding new random energy-minimized conformations to the bank. One round of iterations corresponds to iterations of bank updates until all conformations in the bank are used as seeds. See [1, 2] for more details.
}

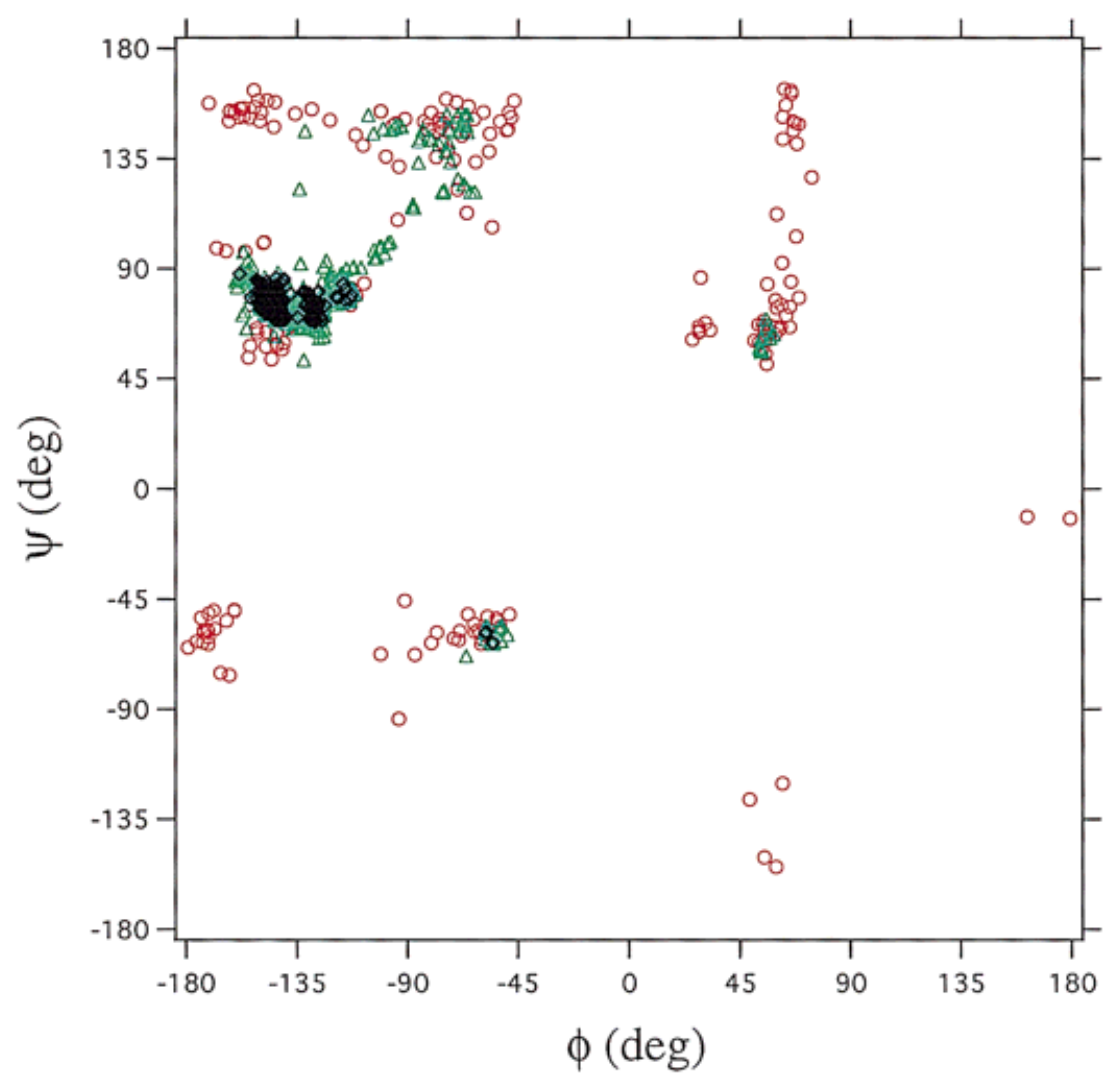

FIGURE 7. The $(\phi, \psi)$ map of the Leu-13 residue of the 20-residue membrane-bound portion of melittin. The red circles were collected from the first bank (randomly generated conformations which were subsequently energy-minimized). The green triangles were collected at an intermediate stage of one CSA run, and the black diamonds were from the bank from which the GMEC was obtained. A similar clustering of data points, as illustrated in Figure 3, is observed. In this peptide the Leu-13 residue precedes Pro-14. 
whole conformational space in its early stages and then narrowing the search to smaller regions of low-energy conformations.

\section{ACKNOWLEDGMENTS}

We thank K. D. Gibson for valuable discussion about parallelization of the CSA. This work was supported by grants from the National Science Foundation (MCB95-13167) and the National Institute of General Medical Sciences (GM-14312) of the National Institutes of Health. The computations were carried out at the Cornell Theory Center which receives funding from Cornell University, New York State, the National Center for Research Resources at the National Institutes of Health, and members of the Center's Corporate Partnership Program.

\section{References}

1. Lee, J.; Scheraga, H. A.; Rackovsky, S. J Comp Chem 1997, $18,1222$.
2. Lee, J.; Scheraga, H. A.; Rackovsky, S. Biopolymers 1998, 46, 103.

3. Momany, F. A.; McGuire, R. F.; Burgess, A. W.; Scheraga, H. A. J Phys Chem 1975, 79, 2361.

4. Némethy, G.; Pottle, M. S.; Scheraga, H. A. J Phys Chem 1983, 87, 1883.

5. Sippl, M. J.; Némethy, G.; Scheraga, H. A. J Phys Chem 1984, 88, 6231.

6. Némethy, G.; Gibson, K. D.; Palmer, K. A.; Yoon, C. N.; Paterlini, G.; Zagari, A.; Rumsey, S.; Scheraga, H. A. J Phys Chem 1992, 96, 6472.

7. Goldberg, D. E. Genetic Algorithms in Search, Optimization, \& Machine Learning; Addison-Wesley: Reading, MA, 1989.

8. Rabow, A. A.; Scheraga, H. A. Pro Sci 1996, 5, 1800.

9. Gay, D. M. ACM Trans Math Software 1983, 9, 503.

10. Ripoll, D. R.; Liwo, A.; Scheraga, H. A. Biopolymers 1998, 46, 117.

11. Pincus, M. R.; Klausner, R. D.; Scheraga, H. A. Proc Natl Acad Sci USA 1987, 79, 5107.

12. Ripoll, D. R.; Scheraga, H. A. Biopolymers 1990, 30, 165.

13. Olszewski, K. A.; Piela, L.; Scheraga, H. A. J Phys Chem 1993, 97, 267.

14. Lee, J.; Liwo, A.; Scheraga, H. A. Proc Natl Acad Sci, USA 1999, 96, 2025.

15. Liwo, A.; Lee, J.; Ripoll, D. R.; Pillardy, J.; Scheraga, H. A. Proc Natl Acad Sci, USA 1999, in press. 